\title{
LES MALADIES DE LA RÉPARATION DE L'ADN : CLONAGE DES GẼNES IMPLIQUÉS DANS LE CONTRÔLE DE L'INTÉGRITÉ DU MATÉRIEL GÉNÉTIQUE
}

\author{
Catherine DIATLOFF-ZITO
}

Institut Curie-Biologie

URA 1292 du CNRS

26 rue d'Ulm, 75231 Paris cedex 05

Les processus qui président au contrôle du métabolisme de l'ADN dans les cellules de mammifères sont des gènes essentiels, les mutations qui les affectent conduisent souvent à des organismes non viables. On s'attend à ce que les maladies dans lesquelles ces processus sont affectés soient rares. En effet, les maladies décrites, consécutives à des mutations touchant par exemple des gènes de la réplication de l'ADN, la transcription ou la ségrégation de chromosome, n'ont pas été décrites (1). Il existe cependant une classe de maladies appelée "maladies de la réparation de l'ADN". Ces principales maladies sont énumérées dans la table 1 . Ces maladies sont rares, transmises de manière autosomique récessive et caractérisées par un défaut ubiquitaire de réparation des lésions de l'ADN.

In vitro, le défaut se manifeste essentiellement par une hypersensibilité spécifique aux radiations et à divers agents chimiques génotoxiques (voir table I). L'instabilité chromosomique est souvent présente. Les principales caractéristiques de ces maladies sont résumées dans la table I (voir pour revue 2,3 ).

La majorité des malades atteints de XP sont déficients dans le système d'excision des nucléotides lésés par les radiations UV; les cellules des malades atteints de CS sont déficientes dans un processus qui assure la réparation préférentielle du brin transcrit de l'ADN dans les gènes actifs ; l'AF démontre une déficience dans l'étape d'incision des pontages et de divers monoadduits de l'ADN; chez AT, les cellules sont sensibles aux RX. La réplication semi-conservative de l'ADN n'est pas bloquée après traitement par $R X$. L'induction par les RX d'un gène qui contrôle l'arrêt du cycle cellulaire est défective (4); BS démontre un ralentissement de la réplication de l'ADN partiellement dû à un disfonctionnement de l'ADN ligase I. 
TABLE I : Maladies humaines caractérisées par une instabilité chromosomique et un défaut de réparation de I'ADN

\begin{tabular}{|c|c|c|c|c|c|}
\hline maladie $\mathrm{a}^{\mathrm{a}}$ & $\begin{array}{l}\text { prédisposition } \\
\text { au cancer }\end{array}$ & $\begin{array}{l}\text { hypersensibilité } \\
\text { de }\end{array}$ & $\begin{array}{l}\text { nbre de groupes } \\
\text { e complémentation }\end{array}$ & $\begin{array}{l}\text { nbre de gènes } \\
\text { clonés }\end{array}$ & $\begin{array}{c}\text { localisation } \\
\text { chromosomique } \\
\text { des gènes }\end{array}$ \\
\hline $\mathrm{XP}$ & cancer de la peau & UV & 7 & 3 & $\begin{array}{c}9 q 34-1 \\
2 q 21 \\
19 q 13-2,13-3\end{array}$ \\
\hline $\mathrm{CS}$ & - & UV & 2 & 1 & $10 q 11-21,1$ \\
\hline $\mathrm{AT}$ & iymphomes & RX & 4 & 0 & $11 q 22-23^{c}$ \\
\hline$A F$ & leucémies & pontages $^{\mathrm{d}}, \mathrm{MA}^{\mathrm{d}}$ & 4 & 1 & $\begin{array}{c}20 q^{c} \\
9 q 22-3\end{array}$ \\
\hline BS & tous types & divers agents & 1 & 0 & - \\
\hline $\mathrm{Rb}$ & $\begin{array}{l}\text { tumeur de l'oeil } \\
\text { ostéosarcome }\end{array}$ & RX & 1 & 1 & $13 q 14$ \\
\hline $\begin{array}{l}\text { XP : xero } \\
\text { syndrome } \\
\text { groupe de } \\
\text { gene non c } \\
\text { pontages e } \\
\text { psoralenes }\end{array}$ & $\begin{array}{l}\text { ma pigmentosum, Cs } \\
\text { Bloom, Rb : rétinobla } \\
\text { nplémentation défini p } \\
\text { í, localisation chromo } \\
\text { IA (monoadditions) } \\
\text { is UVA. }\end{array}$ & $\begin{array}{l}\text { syndrome de Cock } \\
\text { me } \\
\text { formation d'hybrides } \\
\text { mique obtenue par ar } \\
\text { sécutifs au traiternent }\end{array}$ & $\begin{array}{l}\text { cayne, AT : Ataxie télan } \\
\text { s somatiques entre les cell } \\
\text { nalyse de ségrégation } \\
\text { t par des agents pontants }\end{array}$ & $\begin{array}{l}\text { ngiectasie, AF : a } \\
\text { llules de deux mala }\end{array}$ & $\begin{array}{l}\text { Émie de Fanconi, BS } \\
\text { es différents } \\
\text { cine } C \text {, diépoxybutane }\end{array}$ \\
\hline
\end{tabular}

Deux principales approches ont été employées pour le clonage des gènes impliqués dans ces maladies qui sont l'approche génétique et la complémentation fonctionnelle.

- L'approche génétique utilise les méthodes de la génétique inverse ou clonage par positionnement : il s'agit de déterminer d'abord la localisation du gène en exploitant les marqueurs polymorphiques disponibles afin de déterminer celui qui est situé le plus proche du gène (analyse de ségrégation: méthode des Lod Score). Cette approche a permis la localisation d'au moins 2 gènes de 1'AT dans la région 11q22-23 (5) et un gène de l'AF qui appartiendrait au groupe de complémentation génétique A sur le chromosome 20q (6). L'utilisation de cette approche ne peut pas être généralisée à cause de l'importante hétérogénéité génétique dans ces maladies. Dans le cas de l'AF, le marqueur le plus proche sur le chromosome 20 se révèle cependant trop distant du gène pour envisager une marche sur le chromosome (6).

- La seconde alternative possible est la complémentation fonctionnelle des propriétés phénotypiques particulières des cellules des malades, en général l'hypersensibilité à l'action cytotoxique d'une classe d'agents physiques ou chimiques spécifiques. Le gène est alors 
clonable par les méthodes classiques dans les cellules des malades ayant recouvré un phénotype normal (voir pour revue 7).

Dans cette stratégie, le transfert de gène(s) est utilisé pour introduire, dans les cellules des malades, soit sous forme de librairie génomique ou d'ADN complémentaire, soit sous forme d'ADN total, l'information génétique qui permettra la complémentation du défaut génétique. Les cellules subissent ensuite des sélections séquentielles : 1) cellules ayant capté l'ADN transfecté associé à un gène marqueur dominant de résistance à un antibiotique ; 2) critère biologique de complémentation : résistance à un agent physique ou chimique. Dans le meilleur des cas, on obtient des clones ou des pools de cellules ayant recouvré un phénotype normal. Le gène peut ensuite être cloné dans les cellules corrigées en utilisant des sondes, habituellement des séquences répétées, ou un marqueur plasmidique co-transfecté au départ (voir pour revue 7). Cette approche, bien qu'évidente, s'est révélée difficile à mettre en oeuvre tant pour XP, AT qu'AF. Ceci tient essentiellement à deux raisons qui sont la faible capacité des cellules humaines à intégrer de l'ADN exogène et l'instabilité chromosomique de ces cellules. Le gène de XPA a été cloné par cette approche (8). Les problèmes d'intégration de matériel génétique exogène peuvent être contournés en construisant des banques d'ADN complémentaire dans un vecteur d'expression eucaryote se répliquant de manière épisomique (vecteur $\mathrm{EBV}$ ). Cette approche a permis de cloner le gène d'AF du groupe de complémentation C (9) dont la séquence protéique ne contient aucun motif commun à d'autres protéines, la fonction de ce gène est inconnue.

Il existe des mutants de cellules de hamster $\mathrm{CHO}$ ou V79 construits par mutagénèse in vitro et sélectionnés pour leur phénotype les rapprochant de XP, d'AT ou d'AF $(10,11)$. 2 gènes de XP (XPB, XPD) et un gène de CS ont été clonés par complémentation de tels mutants de hamster avec des ADNs humains (voir pour revue 1). D'après la séquence des $\mathrm{ADNc}$, plusieurs domaines fonctionnels putatifs ont été déterminés : ADN hélicase (XPB, $\mathrm{XPD}$ et $\mathrm{CS}$ ), signaux de localisation nucléaire, etc. La fonction de ces protéines n'est pas encore élucidée.

En conclusion, le clonage des gènes impliqués dans ces maladies offre une opportunité pour déterminer le rôle des gènes impliqués dans des étapes clés du métabolisme de l'ADN. Les connaissances acquises devraient permettre à terme d'établir, au plan fondamental, les relations entre le fonctionnement de ces gènes, en relation avec l'environnement et la prédisposition au cancer. La disponibilité des séquences clonées permettra la construction de modèles animaux par inactivation de ces gènes in vivo par recombinaison homologue. Enfin, pour certaines maladies comme l'AF caractérisée par un défaut de différenciation de la moëlle osseuse, la thérapie génique peut être envisagée. 


\section{Références}

1. Hoeijmakers J.H.J. and Bootsma D. (1992) Nature Genetics, 1, 313-314.

2. Friedberg E.C. (1985) DNA Repair (Freeman, New York).

3. Hanawalt P.C. and Sarasin A. (1986) Trends in Genetics, 2, 124-129.

4. Kastan M.B. (1992) Cell, 71, 587-597.

5. Gatti R.A. et al. (1988) Nature, 336, 577-580.

6. Mann W.R. et al. (1991) Genomics, 9, 329-337.

7. Kaina B. et al. (1991) Radiat. Environm. Biophys., 30, 1-19.

8. Tanaka K. et al. (1989) Proc. Natl. Acad. Sci. USA, 86, 5512-5516.

9. Strathdee G. et al. (1992) Nature Genetics, 1, 196-198.

10. Busch D. et al. (1989) Mutagenesis, 4, 349-354.

11. Arwert F. et al. (1991) Cyrogenet. Cell Genet., 56, 23-26. 